\title{
Caracterisation De La Fraction Recyclable Des Dechets Solides De La Commune Territoriale Beni Mellal-Maroc-Zone Villa-
}

\author{
Mechadi Mohamed \\ Mbarki Mohamed \\ Equipe Transdisciplinaire et Sciences Analytique pour le Développement \\ Durable. Département Chimie et Environnement. \\ Faculté des Sciences et Techniques BENI MELLAL. \\ Université Sultan Moulay Slimane. BENI MELLAL-MAROC \\ Chemsi Zakaria \\ Bureau d'Etudes Techniques BUILDING CONSULTING SERVICES \\ SARL AU, CASA LISSASFA - CASABLANCA - MAROC.

\section{Echajia Malika \\ Ennajhi Laila} \\ Equipe Transdisciplinaire et Sciences Analytique pour le Développement \\ Durable. Département Chimie et Environnement. \\ Faculté des Sciences et Techniques BENI MELLAL. \\ Université Sultan Moulay Slimane. BENI MELLAL-MAROC
}

doi: 10.19044/esj.2016.v12n23p253 URL:http://dx.doi.org/10.19044/esj.2016.v12n23p253

\begin{abstract}
Characterization of recyclable solid waste fraction of the villa area in Beni Mellal Moroccan Territorial Commune was appreciated across the weighing parameters refusal, cardboard, paper, packaging, HDPE, PET, glass, metal and tires. The tire parameter was measured twice (February and December 2014) and presented 2,80\% of the monthly average fraction (5,03 tons / month). The rest of the parameters was considered between February and December 2014. The most important results indicate that recyclable waste fraction reached $80,69 \%$ dominated by the Carton $(88,86$ tons or $49,50 \%)$, followed by paper (28,81 tons or $16,05 \%)$. While the less dominant parameter is the glass (0,91 tonnes) with a percentage of $0,51 \%$. Other parameters such as packaging, HDPE, PET and metal present respectively $4,20 \%, 3,44 \%, 3,09 \%$ and $1,09 \%$ of the fraction of 2014 . These results contributed to the classification and quantification of solid waste from the villa area of the city of Beni Mellal.
\end{abstract}


Keywords: Recyclable fraction, solid waste, villa area, Beni Mellal Territorial Commune, MOROCCO

\section{Résumé}

La caractérisation de la fraction recyclable des déchets solides de la zone villa de la Commune Territoriale BENI MELLAL- MAROC a été appréciée à travers le pesage des paramètres Refus, Carton, papier, Emballage, PEHD, PET, Verre, Métal et Pneu. Le paramètre Pneu a été mesuré deux fois (en Février et Décembre 2014) et a présenté 2,80\% de la fraction annuelle (soit 5,03 Tonnes). Le reste des paramètres a été pris en considération entre Février et Décembre 2014. Les résultats les plus importants indiquent que la fraction recyclable des déchets atteint 80,69\% dominée par le Carton (88,86 Tonnes soit 49,50\%) suivi du papier (28,81 Tonnes soit 16,05\%). Tandis que le paramètre le moins dominant est le verre (0,91 Tonnes) avec un pourcentage de 0,51\%. Les autres paramètres tels que l'Emballage, PEHD, PET et Métal représentent respectivement 4,20\%, $3,44 \%, 3,09 \%$ et $1,09 \%$ de la fraction de 2014. Ces résultats ont contribué à la classification des déchets solides de la zone villa de la Ville de BENI MELLAL et de les quantifier.

Mots clés : Fraction recyclable, déchets solides, zone villa, Commune Territoriale BENI MELLAL, MAROC

\section{Introduction}

En matière de gestion, le mot déchet peut être défini de différentes manières selon le type de considération. Dans la littérature, quatre définitions sont proposées : une économique, une juridique, une matérielle et une environnementale (Sané, 2002). Parmi celles-ci, André et Hubert (1997) retiennent les deux premières définitions qui mettent en exergue la valeur économique du déchet et l'enjeu juridique qui entoure sa gestion future (André et Hubert, 1997).

La réduction des déchets joue aussi un rôle capital dans la diminution de la pollution, la conservation des ressources naturelles et, dans beaucoup de cas, l'économie d'énergie (EPA, 2000). Elle peut aussi modifier le changement global du climat causé par les émissions des gaz à effet de serre (ou GES) $\left(\mathrm{CH}_{4}, \mathrm{CO}_{2}\right.$, etc.) et le réchauffement progressif de la terre qui entraînerait un déséquilibre global des écosystèmes (fonte des glace, extinction d'espèces, etc.) (EPA, 2000).

Le développement socio-économique et l'accroissement démographique que connait le Maroc sont accompagnés de l'augmentation de la quantité et la qualité des déchets solides produits essentiellement par les ménages, les industries et les différents établissements. Ces déchets 
représentent des sources importantes de nuisances aussi bien pour l'homme que pour son environnement (El Maguiri et al., 2014).

La gestion efficace des déchets ne peut s’inscrire dans une vision durable que par la connaissance précise de l'évolution des flux de ces rejets et surtout de leur composition. La connaissance des quantités et de la composition des déchets permet d’optimiser le mode de gestion et de promouvoir, éventuellement, la création de filières de valorisation matières. Ceci contribue non seulement à la salubrité de l'environnement des villes (voie publique, périphéries, etc.), mais aussi peut jouer un rôle significatif dans la lutte contre la pauvreté particulièrement dans les Pays en voie de développement (PED) (Buenrostro et Bocco, 2003 ; Aye et Widjaya, 2005 et Zaïri et al., 2004) grâce à la récupération des différents matériaux et leur vente par les récupérateurs.

Les déchets urbains solides sont générés de façon continue en quantité croissante avec le développement des modes de vie des sociétés. Ils sont hétérogènes et leur composition quantitative varie beaucoup en fonction de l'espace (d'une société à l'autre, d'un pays à l'autre, d'une ville à l'autre, etc.) et du temps (jours de la semaine, jours atypiques, fêtes et autres), saisons (humide et sèche, etc.) (Buenrostro et Bocco, 2003). En effet, les facteurs géographique, climatique, économique, racial, culturel social et démographique sont déterminants dans la quantité et la composition des déchets générés par une communauté donnée (Warith et al., 2005 ; Dong et al., 2003 ; Buenrostro et Bocco, 2003 ; Wicker, 2000 ; Abu-Qudais et AbuQdais, 2000 ; Reinhart et McCauley-Bell, 1996 ; Daskalopoulos et al. 1998 ; Thogersen, 1996 et Soclo et al., 1999).

Le choix du nombre de catégories, suivant lesquelles les déchets sont triés, dépend des objectifs de l'étude et des moyens disponibles pour réaliser celle-ci. Toutefois, les principales composantes d'une poubelle ménagère et des déchets urbains solides (DUS) en général restent celles répertoriées par l'ADEME dans le MODECOM en 1993 (MODECOM, 1993) et reprise dans la norme française XP X 30-408 (AFNOR, 1996). Il s'agit des putrescibles, du papier, du carton, des textiles, des textiles sanitaires, des plastiques, des combustibles non classés, des incombustibles non classés, du verre, des métaux, des spéciaux et des fines. Cependant, pour des objectifs spécifiques visés par la caractérisation, certains auteurs se limitent à quelques-unes de ces catégories. Ainsi, Buenrostro et Bocco (2003) ont donné la composition des déchets suivant sept catégories, Mohee (2002) en a défini huit alors que Thogersen (1996) s’est intéressé à l'étude de deux catégories de déchets : les fermentescibles issus des refus de cuisine et les emballages. L'étude de caractérisation des déchets suivants les principales catégories est indispensable dans certains cas où on ne dispose pas de données de référence pour le pays considéré (Aloueimine et al. 2005) ou pour la mise à jour de ces 
données (ADEME, 2005). Il est toutefois important de signaler que la détermination de tous ces paramètres n'est pas toujours nécessaire. Il est souvent suffisant d’analyser un seul groupe spécifique pour répondre à une question donnée sur la gestion des déchets. Par exemple, pour le recyclage matière, l'analyse du groupe matériaux est suffisante alors que l'évaluation de l'impact des émissions de l'incinération sur l'environnement nécessite une analyse de la composition élémentaire des déchets (Brunner et Ernst 1986).

Par ailleurs, la collecte et le transfert constituent la partie la plus visible du système de gestion des déchets solides pour la population en milieu urbain. Ils absorbent une part considérable des budgets municipaux. Dans les pays industrialisés, les frais de collecte représentent 60 à $70 \%$ du total des dépenses, alors que cette proportion dans les Pays en voie de développement dépasse les 70\%. Au Maroc elle varie entre 70 et $90 \%$ de l'ensemble des frais de gestion des déchets solides (DGCL-DEA, 1991). Ainsi, il apparaît clairement que les services de collecte et d'élimination, même dans leur état partiel, coûtent cher aux communes. Ceci remet en cause l'étude de la faisabilité de la valorisation de ces déchets.

Le présent travail s'inscrit dans le cadre de la réalisation d'une étude de faisabilité de mise en place d'un tri sélectif et la valorisation de la fraction recyclable au niveau de la ville de BENI MELLAL. Dans un premier temps, le cadre d'étude comprend la zone villa de la ville de BENI MELLAL, pour englober dans un deuxième temps toute la ville qui a un tonnage moyen des déchets solides et assimilés estimé à 135 tonnes/jour d’après la Commune Territoriale de BENI MELLAL.

L’objectif principal de la présente étude est la mise en exergue de la fraction recyclable des déchets solides produits par la zone villa de la ville de BENI MELLAL.

\section{Matériels et méthodes}

\section{Situation Géographique et Cadre Climatique}

La zone d'étude est une zone villa de la ville de BENI MELLAL. Elle comprend 20000 habitants et faisant partie de 192362 habitants de la ville de BENI MELLAL (B.O.R.M, 2015). (Figure 1) 


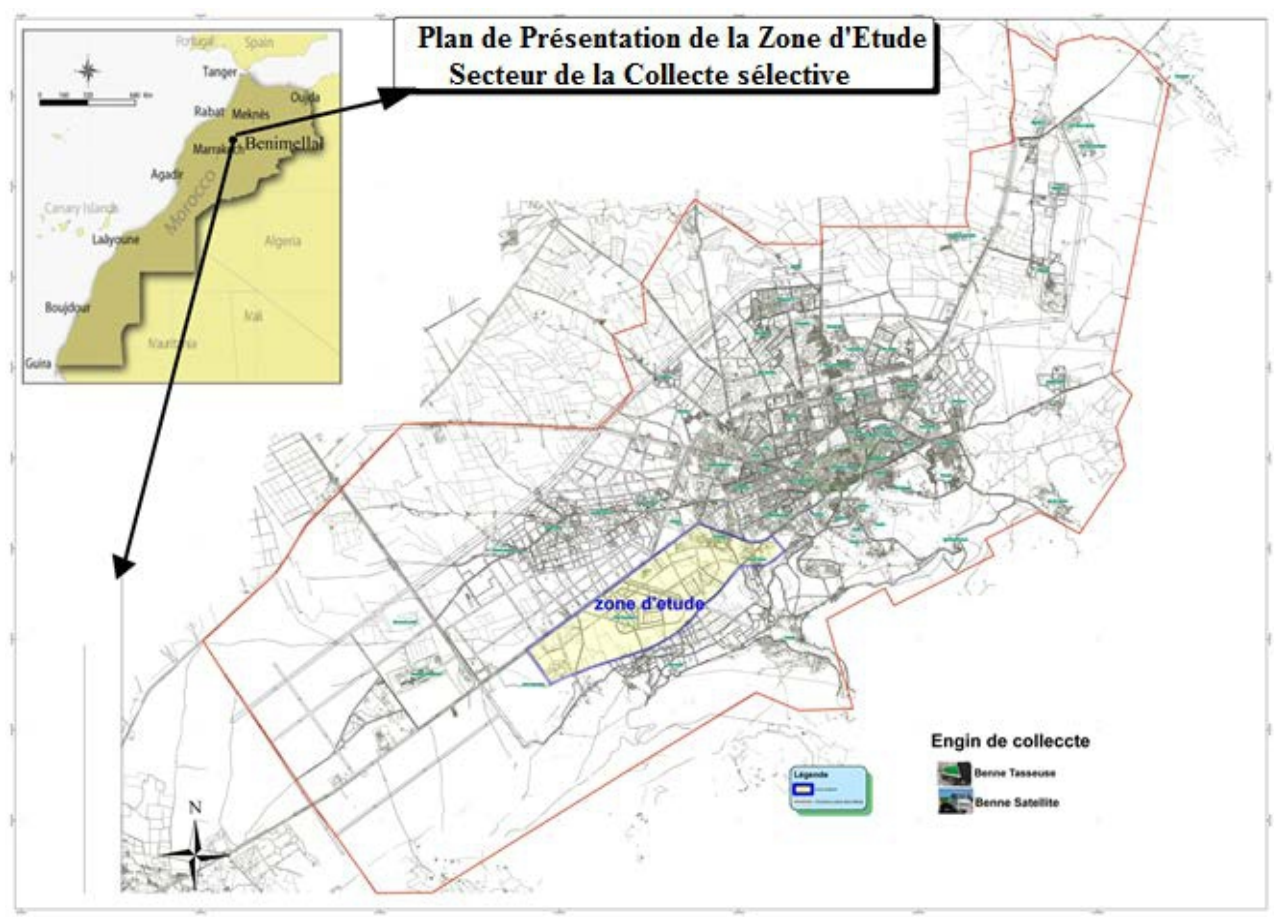

Figure 1: Localisation de la zone d'étude sur le Plan d'aménagement de la ville BENI MELLAL.

Le climat de la région est de type semi-aride avec des hivers tempérés et des étés très chauds. Il est caractérisé par (ABHOER, 2016) :

- Des températures estivales très élevées (moyenne des maxima $37,7^{\circ} \mathrm{C}$ ) et hivernales basses (moyenne des minima $4,9^{\circ} \mathrm{C}$ ).

- $\quad$ Une très forte évaporation. L'évaporation moyenne annuelle est de $1750 \mathrm{~mm}$.

- $\quad$ Une pluviométrie irrégulière de moyenne (550 mm/an).

- $\quad$ les vents dominants sont de secteur Ouest à Nord-Ouest, mais le climat reste influencé par les vents chauds et secs.

Le choix de la zone d'étude est basé sur les critères suivants :

- $\quad$ L'existence de l'espace requis pour les points de collecte ;

- $\quad$ Fréquentation importante par une population assez aisée qui est facile à être sensibilisée, et peut être mobilisée pour participer à la collecte ;

- $\quad$ Existence d'importantes quantités de produits recyclables.

\section{Méthodologie}

Le tri à la source est réalisé par les ménages des zones concernées. Il comporte uniquement la séparation des déchets ménagers en deux fractions : les déchets organiques qui sont acheminés quotidiennement vers les conteneurs classiques implantés dans les rues et les déchets valorisables 
(papier, carton, plastiques, aluminium, fer...) qui sont stockés dans des conteneurs individuelles (ou en point de regroupement) de couleur différente. Un tri complémentaire a, alors, été réalisé dans le centre de tri pour la séparation des différentes fractions des déchets issue de la collecte sélective.

La collecte sélective a été réalisée en collaboration avec le délégataire du service de propreté de la zone d’étude qui est la société Casatechnique, et s’est déroulée comme suit :

- La collecte sélective réalisée en porte à porte en bacs roulants majoritairement (porte à porte et point de regroupement) une fois par semaine (cette fréquence peut aller à une fois par quinze jours selon les saisons et la quantité des déchets valorisables produite) ;

- Le transport des déchets de collecte sélective vers l'exutoire de traitement après son pesage par un pont bascule ;

- Le tri manuel et / ou mécanique des déchets de collecte sélective, puis la classification selon des catégories (carton, papier, plastic, verre...).

La campagne de la collecte sélective a commencé en Février 2014 et a fini en Décembre 2014. Cette campagne concerne la classification des déchets selon huit catégories et deux sous-catégories :

Refus : cette fraction comprend tout élément non recyclé et qui n’appartient à aucune autre catégorie.

Carton : cette fraction est constituée des cartons d'emballages qui regroupent notamment les boîtes de céréales, de riz, de dentifrice, etc. ;

Papier : comprend les journaux et les chutes des papiers ;

Emballage: Les emballages ménagers (hors verre, plastique et métal) intègrent les cartons d'emballages notamment celles du lait ainsi que les briques de jus de fruits et de soupe ;

\section{Plastique qui comprend deux sous-catégories}

PET : PolyEthylène Téréphtalate. Caractérisé par les bouteilles de limonade et autres boissons effervescentes. Elles peuvent être aussi en PVC ou en PC (polycarbonate)

PEHD : Polyéthylène haute densité. Il est utilisé pour produire des caisses en plastique hautement résistantes, emballages de produits détergents, bidons d'huile moteur, bouteilles de shampoing, flacons de médicaments, bouchons de boissons gazeuses, des tubes.

Verre : Le verre est récupéré sous deux formes : i) du verre en débris ou morceaux, ii) du verre sous forme de pièces entières, à savoir les bouteilles de diverses tailles et couleurs.

Métal : le métal est récupéré sous des formes de files, des plaques.

Pneu : cette fraction a été échantillonnée en décembre 2014 et comprend les pneus des voitures. 


\section{Résultats et discussions}

\section{Caractérisation de la fraction recyclable}

La fraction recyclable des déchets solides de la zone Villa de la Commune Territoriale BENI MELLAL a un volume moyen mensuel de 16,318 Tonnes. Elle est caractérisée par le Carton qui présente le tonnage moyen mensuel le plus important de l'ordre de 8,08 Tonnes soit 49,50\% des déchets. Puis, il y a les refus et papier qui présentent respectivement un pourcentage de $19,31 \%$ et $16,05 \%$ avec un tonnage moyen mensuel de 3,15 et 2,62 Tonnes. (Tableau 1)

Les autres types de fraction recyclables des déchets présentent des tonnages moyens mensuels qui ne dépassent pas 0,69 Tonne pour l'Emballage et un tonnage minimal de 0,08 Tonne pour le Verre soit 0,51\% de la fraction recyclable. Aussi, PEHD, PET et Métal présentent respectivement des tonnages moyens mensuels de 0,$56 ; 0,50$ et 0,18 Tonnes. Les Pneus enregistrent un tonnage de 5,03 Tonnes présentant 2,80\% de la fraction recyclable.

La caractérisation de la fraction recyclable révèle, généralement, des valeurs comparables entre l'emballage, le PEHD et le PET et un tonnage global de 179,50 Tonnes en 2014 dont 80,69\% peuvent être valorisées soit 144,84 Tonnes. (Tableau 1)

Tableau 1: Tonnage moyenne, déviation et pourcentage de la Fraction recyclable de la zone d'étude.

\begin{tabular}{|c|c|c|c|c|}
\hline & $\begin{array}{c}\text { Tonnage en } \\
2014(\mathrm{en} \\
\text { Tonnes) }\end{array}$ & Moyenne (en Tonnes/mois) & Déviation & $\begin{array}{c}\text { Pourcentage de } \\
\text { la fraction (\%) }\end{array}$ \\
\hline Refus & $\mathbf{3 4 , 6 6}$ & $\mathbf{3 , 1 5}$ & $\mathbf{0 , 7 8}$ & $\mathbf{1 9 , 3 1}$ \\
\hline Carton & $\mathbf{8 8 , 8 6}$ & $\mathbf{8 , 0 8}$ & $\mathbf{3 , 2 7}$ & $\mathbf{4 9 , 5 0}$ \\
\hline Papier & $\mathbf{2 8 , 8 1}$ & $\mathbf{2 , 6 2}$ & $\mathbf{0 , 6 4}$ & $\mathbf{1 6 , 0 5}$ \\
\hline Emballage & $\mathbf{7 , 5 5}$ & $\mathbf{0 , 6 9}$ & $\mathbf{0 , 1 7}$ & $\mathbf{4 , 2 0}$ \\
\hline PEHD & $\mathbf{6 , 1 8}$ & $\mathbf{0 , 5 6}$ & $\mathbf{0 , 1 4}$ & $\mathbf{3 , 4 4}$ \\
\hline PET & $\mathbf{5 , 5 4}$ & $\mathbf{0 , 5 0}$ & $\mathbf{0 , 1 2}$ & $\mathbf{3 , 0 9}$ \\
\hline Verre & $\mathbf{0 , 9 1}$ & $\mathbf{0 , 0 8}$ & $\mathbf{0 , 0 2}$ & $\mathbf{0 , 5 1}$ \\
\hline Métal & $\mathbf{1 , 9 5}$ & $\mathbf{0 , 1 8}$ & $\mathbf{0 , 0 4}$ & $\mathbf{1 , 0 9}$ \\
\hline Pneus & $\mathbf{5 , 0 3}$ & - & - & $\mathbf{2 , 8 0}$ \\
\hline Total & $\mathbf{1 7 9 , 5 0}$ & $\mathbf{1 6 , 3 2}$ & - & $\mathbf{1 0 0}$ \\
\hline
\end{tabular}

\section{Variation du tonnage de la faction recyclable en 2014 Refus}

Le pesage de cette fraction donne une valeur maximale de 3,92 Tonnes en Septembre et une valeur minimale de 1,43 Tonnes en Février. La variation est indiquent trois augmentations significatives: de 1,43 à 3,83 Tonnes entre Février et Avril, de 2,73 à 3,92 Tonnes entre Juillet et Août et de 3,37 à 3,61 Tonnes entre Octobre et Décembre (Figure 2). 
Généralement, le tonnage est comparable en Mai (21,38 Tonnes), Juin (20,70Tonnes), Juillet (20,16 Tonnes), Octobre (20,96 Tonnes) et Novembre (20,05 Tonnes). Aussi, en mois d'Août (14,10 Tonnes) et Décembre (13,66 Tonnes). (Figure 2)

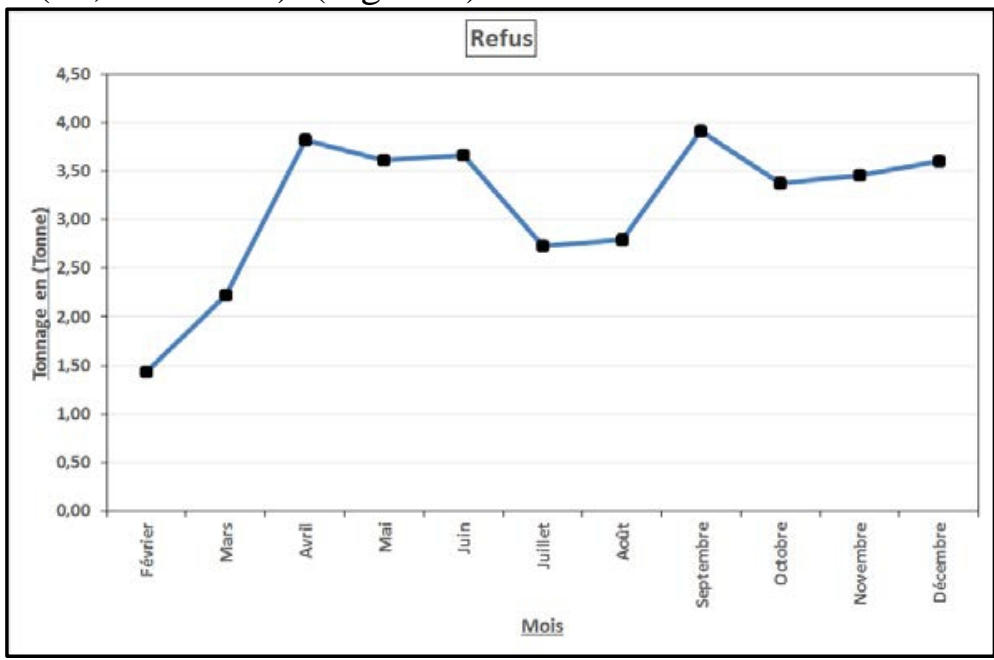

Figure 2: Diagramme de variation du tonnage moyen mensuel des Refus en 2014.

\section{Carton}

La variation du tonnage du Carton est trimestrielle ; elle a enregistré une augmentation de la valeur minimale 4,91 à 8,66 Tonnes entre Avril et Juin, puis une diminution de la valeur maximale 12,93 à 7,76 Tonnes entre Août et Octobre pour rebondir à 12,48 Tonnes en Décembre. (Figure 3)

Le Tonnage est comparable en Mai (7,62 Tonnes), Juillet (7,62 Tonnes), Octobre (7,76 Tonnes). Il est comparable, aussi, entre le Tonnage des mois Juin (8,66 Tonnes), Novembre (8,35 Tonnes) et entre Août (12,93 Tonnes) et Décembre (12,48 Tonnes). (Figure 3)

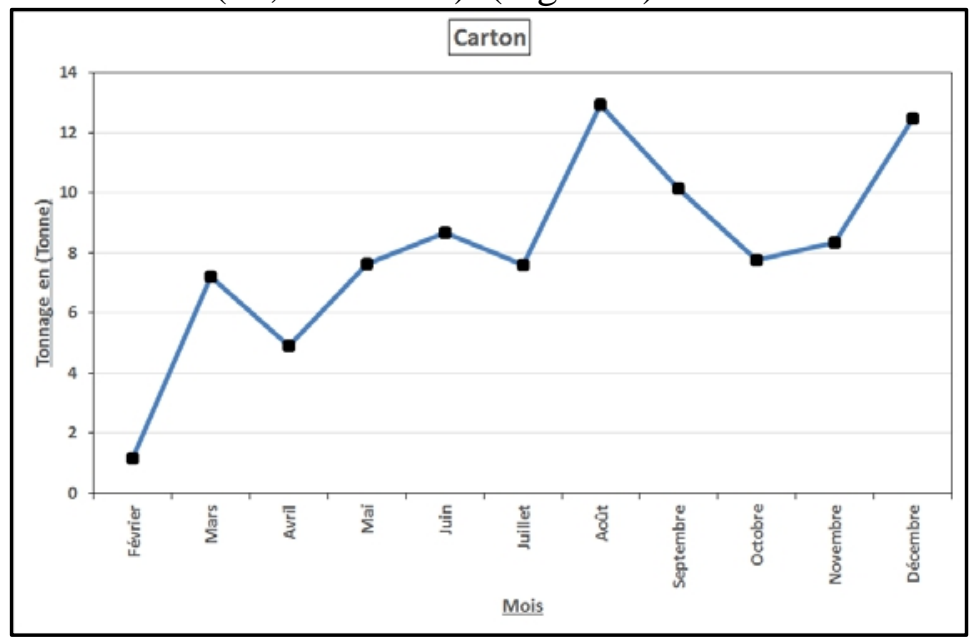

Figure 3: Diagramme de variation du tonnage moyen mensuel du Carton en 2014. 


\section{Papier}

Le tonnage du Papier dans la zone d'étude a enregistré trois augmentations. La première est très significative; d'une valeur minimale 1,19 à 3,18 Tonnes entre Février et Avril. Une deuxième augmentation est progressive de 2,27 à une valeur maximale de 3,26 tonnes entre Juillet et Septembre. La dernière augmentation est linéaire de 2,80 à 3 Tonnes entre Octobre et Décembre. (Figure 4)

La masse de la fraction papier est comparable entre les mois d'Avril (3,18 Tonnes) et Septembre (3,26 Tonnes) et entre les mois de Mai (3,01 Tonnes), Juin (3,05 Tonnes) et Décembre (3,00 Tonnes). Entre les mois de Juillet (2,27 Tonnes) et Août (2,32 Tonnes). Et entre Octobre (2,80 Tonnes) et Novembre (2,88 Tonnes). (Figure 4)

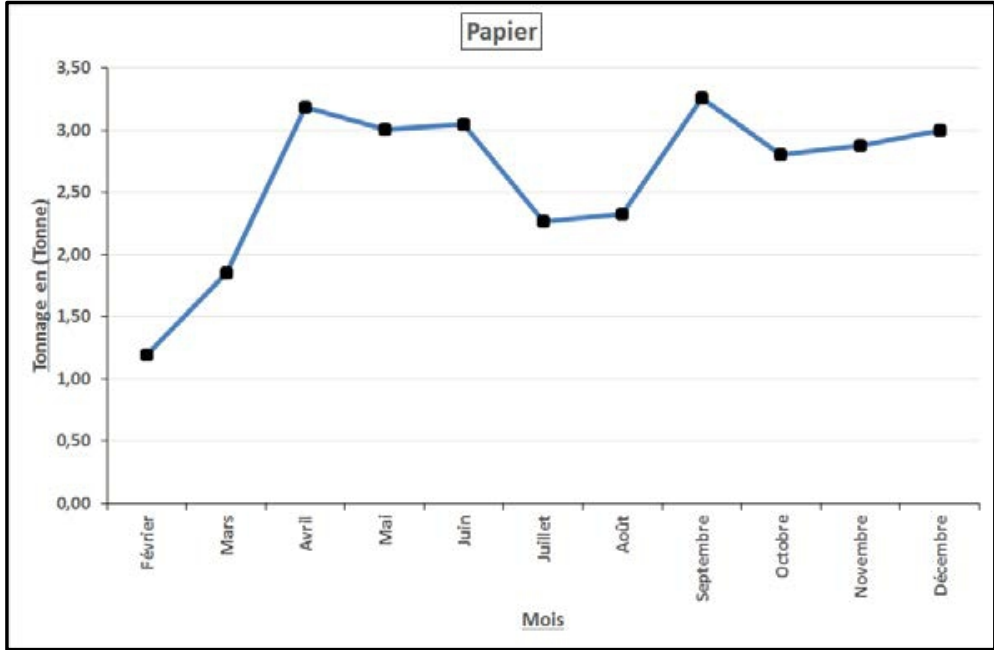

Figure 4: Diagramme de variation du tonnage moyen mensuel du Papier en 2014.

\section{Emballage}

Le tonnage de l'Emballage dans la zone d'étude a enregistré trois augmentations. La première varie d'une valeur minimale 0,31 à 0,83 Tonne entre Février et Avril. Une deuxième augmentation est progressive de 0,59 à une valeur maximale de 0,85 tonne entre Juillet et Septembre. La dernière augmentation connait une légère variation de 0,73 à 0,79 Tonne entre Octobre et Décembre. (Figure 5)

La masse de la fraction Emballage est comparable entre les mois d'Avril (0,83 Tonne) et Septembre (0,85 Tonne), entre les mois de Mai (0,79 Tonne), Juin (0,80 Tonne) et Décembre (0,79 Tonne). Entre les mois de Juillet (0,59 Tonne) et Août (0,61 Tonne). Et entre Octobre (0,73 Tonne) et Novembre (0,75 Tonne). (Figure 5) 


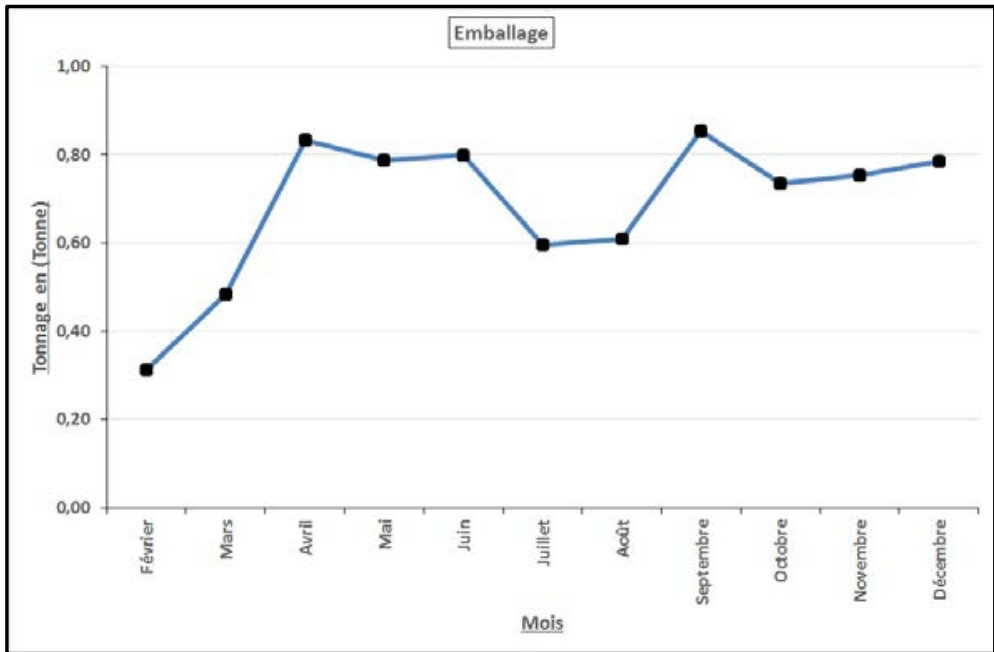

Figure 5: Diagramme de variation du tonnage moyen mensuel de l'Emballage en 2014.

\section{PEHD}

Le tonnage du PEHD a donné trois augmentations. La première varie d’une valeur minimale 0,26 à 0,68 Tonne entre Février et Avril. Une deuxième augmentation est progressive de 0,49 à une valeur maximale de 0,70 tonne entre Juillet et Septembre. La dernière augmentation connait une légère variation de 0,60 à 0,64 Tonne entre Octobre et Décembre. (Figure 6)

La masse de la fraction PEHD est comparable entre les mois d'Avril (0,68 Tonne) et Septembre (0,70 Tonne), entre les mois de Mai et Juin (0,65 Tonne) et Décembre (0,64 Tonne). Entre les mois de Juillet (0,49 Tonne) et Août (0,50 Tonne). Et entre Octobre (0,60 Tonne) et Novembre $(0,62$ Tonne). (Figure 6)

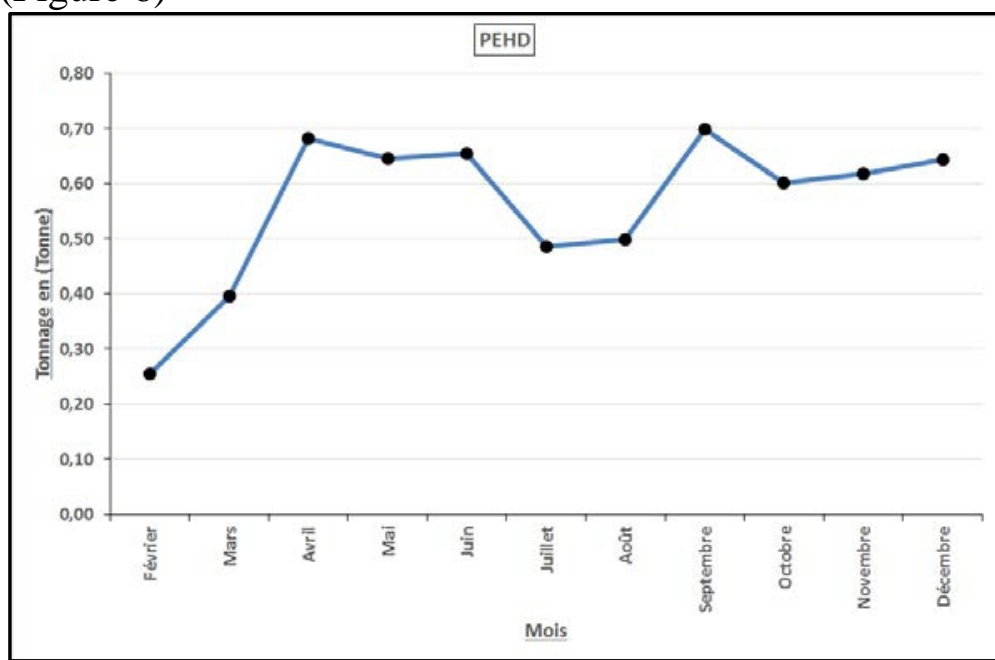

Figure 6: Diagramme de variation du tonnage moyen mensuel de PEHD en 2014. 


\section{PET}

Trois augmentations sont enregistrées pour le PET. La première varie d'une valeur minimale 0,23 à 0,61 Tonne entre Février et Avril. Une deuxième augmentation est progressive de 0,44 à une valeur maximale de 0,63 tonne entre Juillet et Septembre. La dernière augmentation connait une légère variation de 0,54 à 0,58 Tonne entre Octobre et Décembre. (Figure 7)

La masse de la fraction PEHD est comparable entre les mois d'Avril (0,61 Tonne) et Septembre (0,63 Tonne), entre les mois de Mai et décembre (0,58 Tonne) et Juin (0,59 Tonne). Entre les mois de Juillet (0,44 Tonne) et Août $(0,45$ Tonne). Et entre Octobre (0,54 Tonne) et Novembre (0,55 Tonne). (Figure 7)

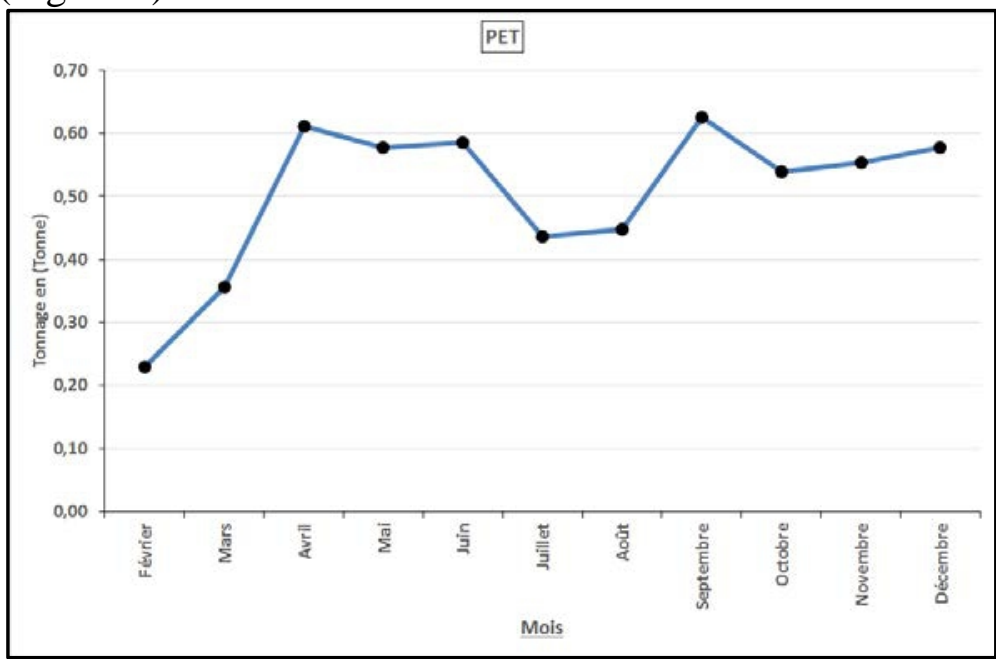

Figure 7: Diagramme de variation du tonnage moyen mensuel (en Tonne) de PET en 2014.

\section{Verre}

Le Tonnage du Verre varie légèrement entre 0,04 Tonne en Février 0,10 Tonne en Avril, Juin et Septembre (Figure 8). Le tonnage enregistre des valeurs similaires de 0,07 Tonne en Juillet et Août, et de 0,09 Tonne en Mai, Octobre, Novembre et Décembre. Des valeurs similaires, aussi, de 0,10 Tonne enregistrées en Avril, Juin et Septembre. 


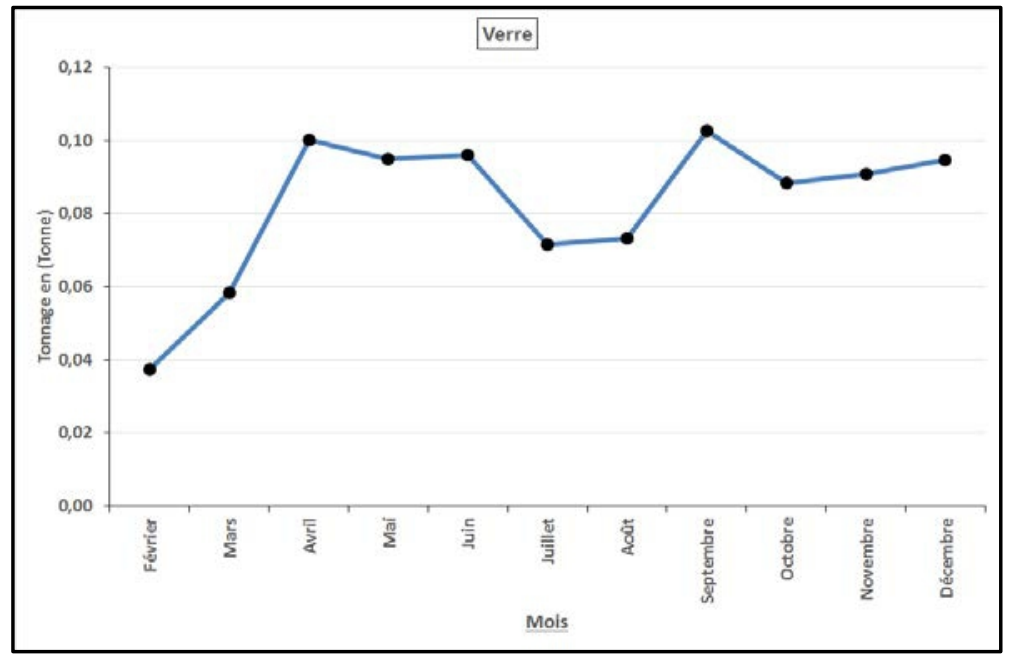

Figure 8: Diagramme de variation du tonnage moyen mensuel du Verre (en Tonne) en 2014.

\section{Métal}

Les résultats de la fraction du métal montrent qu'elle varie entre 0,08 Tonne en Février et 0,22 Tonne enregistrée en Avril et Septembre.

Les résultats montrent, aussi, deux augmentations ; de 0,08 à 0,22 Tonne entre Février et avril et de 0,15 à 0,22 Tonne entre Juillet et Septembre.

Des valeurs similaires sont enregistrées, de 0,22 Tonne en Avril et Septembre, et de 0,20 Tonne en Mai, Novembre et Décembre.

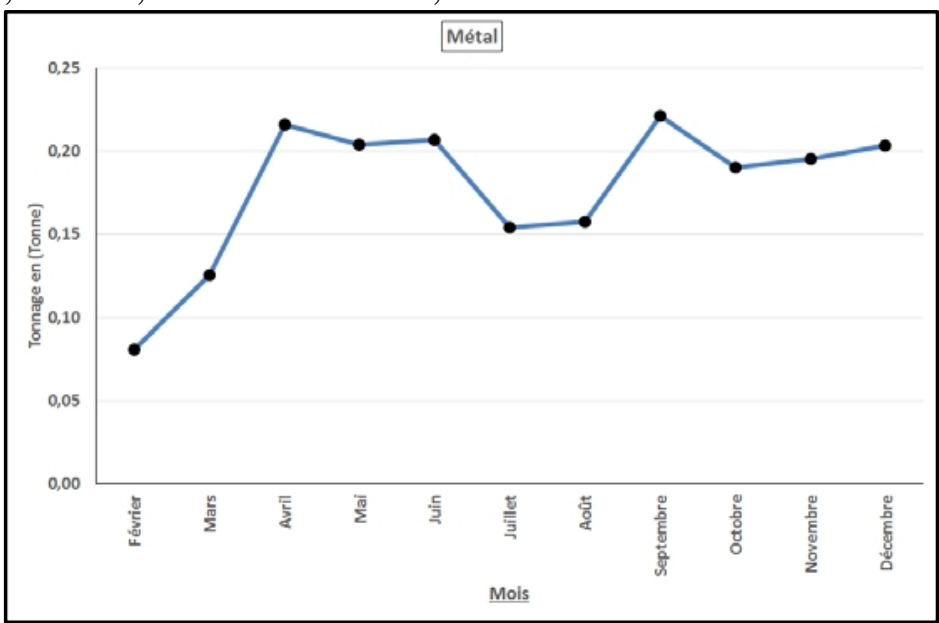

Figure 9: Diagramme de variation du tonnage moyen mensuel du Métal (en Tonne) en 2014.

\section{Pneu}

Cette fraction qui a connu deux campagnes d'échantillonnage est de l'ordre de 5,03 tonnes. 


\section{Analyse statistique}

L'analyse statistique, de la fraction recyclable des déchets solides de la zone villa de la ville de Béni mellal par le Test de Pearson, a montré (Tableau 2) :

- $\quad$ Qu’il n’y a pas de corrélations significatives entre le Carton et le reste de la fraction recyclable ;

- Qu’il y a des corrélations très significatives entre le reste de la fraction recyclable ;

- Que les corrélations entre la fraction Refus, Papier, Emballage, PEHD et PET sont très significatives $(\mathrm{P}=1)$;

- $\quad$ Que la corrélation entre le verre et le métal et le reste de la fraction recyclable est très significative $(0,989 \leq \mathrm{P} \leq 0,997)$.

Tableau 2: Corrélation de Pearson entre les fractions recyclables des déchets solides de la zone d'étude

\begin{tabular}{cccccccc}
\hline & Refus & Carton & Papier & Emballage & PEHD & PET & Verre \\
\hline Carton & 0,503 & & & & & & \\
Papier & $\mathbf{1 , 0 0 0}$ & 0,501 & & & & & \\
Emballage & $\mathbf{1 , 0 0 0}$ & 0,508 & $\mathbf{1 , 0 0 0}$ & & & & \\
PEHD & $\mathbf{1 , 0 0 0}$ & 0,500 & $\mathbf{1 , 0 0 0}$ & $\mathbf{1 , 0 0 0}$ & & & \\
PET & $\mathbf{1 , 0 0 0}$ & 0,511 & $\mathbf{1 , 0 0 0}$ & $\mathbf{1 , 0 0 0}$ & $\mathbf{1 , 0 0 0}$ & & \\
Verre & $\mathbf{0 , 9 9 0}$ & 0,439 & $\mathbf{0 , 9 9 0}$ & $\mathbf{0 , 9 8 9}$ & $\mathbf{0 , 9 8 9}$ & $\mathbf{0 , 9 9 0}$ & \\
Métal & $\mathbf{0 , 9 9 7}$ & 0,493 & $\mathbf{0 , 9 9 7}$ & $\mathbf{0 , 9 9 6}$ & $\mathbf{0 , 9 9 6}$ & $\mathbf{0 , 9 9 6}$ & $\mathbf{0 , 9 9 3}$ \\
\hline
\end{tabular}

\section{Composition moyenne annuelle par habitant de la fraction recyclable}

La composition de la fraction recyclable montre que l'habitant de la zone d'étude (Zone villa) de la ville de BENI MELLAL produit 9,79Kg/hab.an de fraction recyclable dont presque la moitié est caractérisée par le Carton $(4,85 \mathrm{~kg} / \mathrm{hab} . \mathrm{an})$. Le reste de la fraction est caractérisé par la production de 1,57 kg/hab.an de papier, de 0,41 kg/hab.an d'Emballage, de 0,64 kg/hab.an de Plastique (PEHD et PET), de 0,05 kg/hab.an du Verre, de $0,11 \mathrm{~kg} / \mathrm{hab} . a n$ du métal et $0,27 \mathrm{~kg} / \mathrm{hab}$.an en Pneu. Or, la production de Refus qui ne sont pas valorisable est de 1,89 kg/hab.an. (Tableau 3)

La comparaison de cette composition avec celle d'un pays développé tel que la France et un pays en développement tel que la Tunisie, montre que l'habitant de la zone villa de la ville de BENI MELLAL produit moins de fraction recyclable que celle de la Tunisie (Ben Ammar, 2006) et de la France (ADEME, 1999) (Tableau 3). La comparaison est comme suit (Tableau 3) :

- $\quad$ 4,85 kg de Carton contre $21 \mathrm{~kg}$ pour la Tunisie (soit 4 fois moins) et $39 \mathrm{~kg}$ pour la France (soit 8 fois mois) ;

- $\quad 1,57 \mathrm{~kg}$ de Papier contre $22 \mathrm{~kg}$ (soit 14 fois moins) pour la Tunisie et $67 \mathrm{~kg}$ (soit 42 fois moins) pour la France ; 
- $\quad$ 0,64 kg de Plastique contre $12 \mathrm{~kg}$ (soit 18 fois moins) pour la Tunisie et $46 \mathrm{Kg}$ pour la France ;

- $\quad 0,05 \mathrm{~kg}$ de verre contre $5 \mathrm{~kg}$ pour la Tunisie et $54 \mathrm{~kg}$ pour la France ;

- $\quad 0,11 \mathrm{~kg}$ de Métal contre $7 \mathrm{~kg}$ pour la Tunisie et $17 \mathrm{~kg}$ pour la France.

Tableau 3: Comparaison de la Composition annuelle moyenne par habitant (en kg/hab.an) de la fraction recyclable de la zone villa de la ville de BENI MELLAL avec celle de la Tunisie

(Ben Ammar, 2006) et la France (ADEME, 1999).

\begin{tabular}{cccc}
\hline Fraction & Zone d'étude & Tunisie $^{(3)}$ & France $^{(4)}$ \\
\hline Refus & 1,891 & - & - \\
\hline Carton & 4,847 & 21 & 39 \\
\hline Papier & 1,572 & 22 & 67 \\
\hline Emballage & 0,412 & - & - \\
\hline Plastique & 0,64 & 12 & 46 \\
\hline Verre & 0,050 & 5 & 54 \\
\hline Métal & 0,107 & 7 & 17 \\
\hline Pneus & 0,274 & - & -
\end{tabular}

\section{Discussion}

Nos résultats ont montré qu'il n'y a pas de corrélation entre le carton et le reste des fractions recyclables. Ces dernières qui enregistrent une variation similaire entre elle. En outre, la variation de la fraction recyclable ne tient pas compte des saisons et des périodes de vacances.

La comparaison des fractions recyclables de la zone d'étude avec celles de Québec (Bilan sur la gestion des déchets au Québec, 2002), et l’Université Hassan II-Mohammedia (El maguiri et al. 2014) a montré que la fraction recyclable de la zone d'étude (Tableau 4) :

- $\quad$ En Carton (49,50\%) et Papier (16,05\%) est inférieure à celle de Québec (84,80\%) et supérieure à celle de l'Université Hassan IIMohammedia (9,70\% en Carton et 10,80 en Papier);

- $\quad$ En PET et PEHD (6,53\%) est supérieure à celle de Québec (3,10\%) et inférieure à celle de l'Université Hassan II-Mohammedia $(15,40 \%)$;

- $\quad$ En verre $(0,51 \%)$ est supérieure à celle de Québec $(8,50 \%)$ et inférieure à celle de l'Université Hassan II-Mohammedia (0,30\%) ;

- $\quad$ En métal (1,09\%) est inférieure à celle de Québec (3,70\%) et l’Université Hassan II-Mohammedia. 
Tableau 4: Comparaison des fractions recyclable de la zone d'étude avec celles Québec (Bilan sur la gestion des déchets au Québec, 2002) et l'Université Hassan II-Mohammedia (El maguiri et al. 2014) (en \%)

\begin{tabular}{|c|c|c|c|c|}
\hline Fractions & \multicolumn{2}{|c|}{ Zone d'étude } & Québec $^{(1)}$ & $\begin{array}{c}\text { Université Hassan II- } \\
\text { Mohammedia }^{(2)}\end{array}$ \\
\hline Refus & \multicolumn{2}{|c|}{19,31} & - & - \\
\hline Carton & 49,50 & \multirow{2}{*}{65,55} & \multirow{2}{*}{84,80} & 9,70 \\
\hline Papier & 16,05 & & & 10,80 \\
\hline Emballage & \multicolumn{2}{|c|}{4,20} & - & - \\
\hline PET & 3,09 & \multirow{2}{*}{6,53} & \multirow{2}{*}{3,10} & \multirow{2}{*}{15,40} \\
\hline PEHD & 3,44 & & & \\
\hline Verre & \multicolumn{2}{|c|}{0,51} & 8,50 & 0,30 \\
\hline Métal & \multicolumn{2}{|c|}{1,09} & 3,70 & 2,50 \\
\hline Pneus & \multicolumn{2}{|c|}{2,80} & - & - \\
\hline
\end{tabular}

${ }^{(1)}$ Bilan sur la gestion des déchets au Québec, $2002 ;{ }^{(2)}$ El maguiri et al. 2014

\section{Conclusion}

La caractérisation de la fraction recyclable des déchets solides de la zone d'étude permet de conclure que :

- $\quad$ La production moyenne mensuelle des déchets est de 16,32 Tonnes répartie en 8,08 Tonnes de Carton, de 2,62 Tonnes de Papier, de 0,69 Tonne d'Emballage, 0,56 Tonne de PEHD, 0,50 Tonne de PET, 0,18 Tonne de Métal et 0,08 Tonne de Verre ;

- $\quad$ La variation de la fraction recyclable ne tient pas compte des saisons et des périodes de vacances ;

- La composition de la fraction recyclable de la zone d'étude est importante en Carton (49,50\%) et faible en verre $(0,51 \%)$ et métal $(1,09 \%)$;

- $\quad$ L'habitant de la zone d'étude (Zone villa) de la ville de BENI MELLAL produit $9,79 \mathrm{Kg} / \mathrm{hab}$.an de fraction recyclable dont presque la moitié est caractérisée par le Carton (4,85 kg/hab.an). Le reste de la fraction varie entre 1,57 et $0,05 \mathrm{~kg} / \mathrm{hab} . \mathrm{an}$. Tandis que la production de Refus qui ne sont pas valorisable est de $1,89 \mathrm{~kg} / \mathrm{hab} . \mathrm{an}$.

La comparaison de la composition de la fraction recyclable de la zone d'étude avec d'autres zones a permis de conclure que la production de déchets recyclables est très faible dans la zone villa de la ville de BENI MELLAL.

\section{References :}

ABHOER - Agence du Bassin Hydraulique Oum Er Rbia, 2016.

Abu-Qudais M. \& Abu-Qdais H.A. 2000. Energy content of municipal solid waste in Jordan an dits potential utilization; Energy Conversion \& Management 41 (2000) 983 - 991.

ADEME 1999. AGENCE DE L'ENVIRONNEMENT ET DE LA MAITRISE DE L'ENERGIE, FRANCE - La composition des ordures 
ménagères en France - édité par l’Ademe Centre d’Angers, (1 ${ }^{\text {ère }}$ édition) Janvier 1999 - Coll., « Données et Références ». 59p.

ADEME 2000. Atlas des déchets en France $2^{\text {ème }}$ édition (données et références), ADEME éditions, Paris, 138 pages.

AFNOR, 1996. Déchets : Caractérisation d'un échantillon de déchets ménagers et assimilés ; Eds AFNOR ; 24 pages.

Aloueimine S., Matejka G., Zurbrugg C. \& Sidi Mohamed M.E. 2005. Caractérisation des Ordures Ménagers à Nouakchott : Partie II : Résultats en Saison Sèche et en Saison Humide, article en Presse, 8 pages.

André M. \& Hubert S. 1997. Gestion des déchets hospitaliers, Projet DESS ‘'TBH', UTC, pp 51.

Aye L. et Widjaya E.R. 2005. Environmental and economic analysis of waste disposal options for traditional markets in Indonesia, Waste Management xxx (2005) xxx - xxx, Article in Press, accepted 15 September 2005.

Ben Ammar Samira. (2006). Les enjeux de la caractérisation des déchets ménagers pour le choix de traitement adaptés dans les pays en développement - Résultats de la caractérisation dans le Grand Tunis-Mise au point d'une méthode adaptée. Thèse de doctorat, Institut National Polytechnique de Lorraine-Ecole Nationale Supérieure de Géologie de NANCY.

B.O.R.M 2015. Bulletin Officiel du Royaume du MAROC n6354 ISSN 0851 - 1195 (23 Avril 2015) p.4050.

Brunner P. H. \&Walter R. Ernst 1986. Alternative Methods for the Analysis of Municipal Solid Waste, Waste Management \& research (1986) 4, 147160 .

Buenrostro O. et Bocco G. (2003). Solid waste management in municipalities in Mexico: Goal and perspectives, Resources, Conservation and Recycling 39 (2003) 251 - 263.

Daskalopoulos E., Badr O. \& Probert S.D. 1998. Municipal solid waste : a prediction methodology for the generation rate and composition in the European Union countries and the United States of America, Resources, conservation and Recycling 24 (1998) 155 - 166.

DGCL-DEA : DIRECTION GENERALE DES COLLECTIVITES LOCALES - DIRECTION DE L'EAU ET DE L'ASSAINISSEMENT, MAROC (1991) - Principes et pratiques pour la gestion rationnelle des déchets solides municipaux - Document réalisé pour le Ministère de l'Intérieur Marocain avec le soutien de l'Agence Américaine pour le Développement International (USAID), 1991, 82p.

Dong, C., Jin B. \& Li, D. 2003. Predicting the heating value of MSW with a feed forward, neural network, Waste Management 23 (2003) 103 - 106. 
El maguiri Abdelhakim, Idrissi Laila, Abouri Meriem, Souabi Salah, Taleb Abdeslam, Youbi Redouan 2014. Etude de mise en place d'un tri séléctif à l’Université de Mohammedia, Maroc. Déchets Sciences et Techniques (Juin 2014), p $12-19$.

EPA, 2000. Reducing Waste Can Make a World of Difference: The Link Between Solid Waste and Global Climate Change, Reusable News, EPA 530-N-00-06 Fall 2000, Environmental Protection Agency, Emergency Response (5305W), 12 pages.

MODECOM 1993. Méthode de Caractérisation des Ordures Ménagères/ $2^{\text {ème }}$ édition, ADEME éditions, Paris, 64 pages.

Mohee R. 2002. Assessing the recovery potential of solid waste in Mauritius; Resources, conservation and Recycling 36 (2002) 33 - 43.

Rapport, (2004). Statewide Waste Characterization Study, Contractor's Report to the Board, Cascadia Consulting Group, Inc., 124 pages.

Reinhart D.R. et McCauley-Bell P. (1996). Methodology for Conducting Composition Study for Discarded Solid Waste; Florida Center for Solid and Hazardous Waste Management, 82 pages.

Sané Y. 2002. La gestion des déchets à Abidjan : un problème récurrent et apparemment sans solution ; AJEAM/RAGEE 2002 ; Vol. $4 \mathrm{~N}^{\circ} 1$; 13-22

Soclo H. H., Aguewe M., Adjahossou B.C. \& Houngue Th (1999). Recherche de compost type et toxicité résiduelle au Bénin, TSM $\mathrm{N}^{\circ} 9-$ septembre 1999 - 94ème année, 9 pages.

Thogersen J., (1999). Wasteful Food Consumption: Trends In Food And Packaging Waste, Scandinavian Journal of Management, Volume 12, Issue 3, Pages 291-304.

Warith M., Li X. and Jin H., (2005). Bioreactor Landfills: State-of-the-Art Review; Emirates Journal for Engineering Research, 10 (1), 1-14 (2005).

Zaïri M., Ferchichi M., Ismaïl A., Jenayeh M. \& Hammami H. (2004). Rehabilitation of Yahoudia dumping site, Tunisia, Waste Management 24 (2004) 1023 - 1034.

Wicker A. (2000). Chapitre 22: Gestion des Déchets dans « Statistiques pour la politique de l'environnement », 27-28 novembre, Munich ; 12 pages. 